Ann. Biol. anim. Bioch. Biophys., 1979, 19 (3 B), 889-893.

\title{
Analyse automatique du comportement alimentaire et mérycique chez le mouton
}

par M. LEVEILLÉ, J. P. JOUANY, J. P. BRUN*

Laboratoire de la Digestion des Ruminants

* Laboratoire de la Production ovine

I.N.R.A., Theix, Saint-Genès-Champanelle,

63110 Beaumont.

Summary. Automatic analysis of feeding and ruminating behavior in sheep.

Feeding behavior is generally measured by mechanical devices which graphically record the jaw movements (Ruckebusch and Bueno, 1972 ; Ruckebusch, Bueno and Latour, 1973 ; Bechet, 1978). Data-reading is long and does not furnish all the information available. We propose an electronic fechnique which is more accurate and flexible and can be applied in many different ways. The movements of the lower jaw of sheep were detected with a variable inductance gauge composed of a $20 \mathrm{~cm}$ long solenoïd, made from a copper wire (diameter $=0.04 \mathrm{~mm}$ ) wound around a rubber tube $5 \mathrm{~mm}$ in diameter. This unit was flattened and then covered with a polymer to protect it before placing it around the halter noseband of the animal. This transducer was connected to an electronic unit distinguishing the electric signals corresponding to ingestion from those of rumination. The ruminating periods had to be recognized by the following concomitant criteria : a) the time separating 2 cycles of maxillary activity had to exceed 3 seconds $; b$ ) each cycle had to include a number of jaw movements ranging from a minimum of 16 to a maximum of $128 ; c)$ both $(a)$ and $(b)$ had to occur at least twice in succession. The electric impulses identified by the computer were then conveyed to the memory bank which was consulted whenever necessary by means of a digital system. The number of jaw movements during ruminating or feeding periods was thus directly known after being determined by the device. With this equipment we obtained the duration of rest and ruminating or feeding periods throughout the day, as well as the starting and ending of each of these periods. Moreover, this equipment could be used on grazing animals by taking long-distance hertzian readings.

\section{Introduction.}

L'étude du comportement alimentaire ef mérycique des ruminants en stabulation libre ou entravée a fait l'objet, au cours des 15 dernières années, de publications dans lesquelles différentes techniques de mesure ont été proposées. Ainsi Ruckebusch (1963) puis Ruckebusch et Bueno (1972) ont décrit un appareillage simple à mettre en cuvre qui s'adapte particulièrement bien aux animaux maintenus en stabulation entravée. Dans la plupart des méthodes utilisées actuellement, le capteur se compose d'une poire en caoutchouc fixée sur la base rigide d'un licol. Le système d'enregistrement, constitué 
autrefois d'un tambour à levier de Marey, a évolué pour devenir plus léger. L'utilisation d'un vibrographe modifié dont l'encombrement est réduit permet son emploi sur des animaux au pâturage (Ruckebusch et Bueno, 1973 ; Ruckebusch, Bueno et Latour, 1973 ; Bechet, 1978). Cependant, ces appareils fournissent un enregistrement graphique qui nécessite ensuite une analyse manuelle délicate et fastidieuse.

Le but de ce travail a été de mettre au point un matériel léger, basé sur l'exploitafion d'un signal électrique issu d'un capteur simple et original qui permet d'obtenir un plus grand nombre de données pouvant être traitées automatiquement.

\section{Description de l'appareil.}

Capteur de déplacement (fig. 1A). - Les mouvements de la mâchoire inférieure du mouton sont détectés à l'aide d'un capteur de déplacement à inductance variable. Celui-ci est constitué d'un solénoïde à spires jointives de $25 \mathrm{~cm}$ de long, réalisé à l'aide d'un fil de cuivre de $0,04 \mathrm{~mm}$ de diamètre bobiné autour d'un tube en caoutchouc souple de $5 \mathrm{~mm}$ de diamètre préalablement enduit d'un adhésif. L'ensemble est ensuite aplati et peut être enrobé d'un plastique réticulable à l'air, avant d'être fixé autour de la muserole d'un licol porté par l'animal. Chaque déplacement de la mâchoire inférieure correspondant à l'ouverture de la bouche provoque un allongement du solénoïde, donc une modification de son inductance qui est transformée en une variation de fréquence, convertie ensuite en une variation de tension par un convertisseur.

Appareil de mesure (fig. 2B). - Le signal électrique résultant d'un mouvement de mâchoire de l'animal est dirigé vers différentes voies d'analyse grâce à un calculateur logique qui peut distinguer, ovec une probabilité d'erreur de l'ordre de 1 p. 100 , les signaux correspondant respectivement à une prise d'aliment ou à une activité mérycique de l'animal. Pour cela, l'unité centrale de calcul est programmée suivant plusieurs critères qui ont été préalablement déterminés à partir d'une analyse manuelle d'enregistrements graphiques du comportement alimentaire et mérycique. Ces enregistrements ont été effectués sur 5 moutons maintenus en stabulation entravée, qui ont reçu chacun successivement un régime de foin de graminées, de luzerne déshydratée présentée sous forme agglomérée, d'herbe verte, de betteraves et enfin d'aliment concentré à base d'orge. Les paramètres choisis qui permettent au mieux d'identifier une période de rumination sont les suivants : 1) définition des temps séparant 2 cycles, 2) nombre de coups de mâchoire par cycle mérycique, 3) reproductibilité du phénomène observé. Ainsi les phases de rumination sont reconnues par l'analyseur si l'ensemble des conditions suivantes sont satisfaites simultanément : a) le temps séparant 2 cycles d'activité masticatoire doit être supérieur à $3 \mathrm{sec}, b$ ) chaque cycle doit comporter un nombre de coups de mâchoire compris entre un minimum (16) ef un maximum (128), c) chacune des conditions $a$ et $b$ doit se reproduire au moins deux fois

FIG. 1. - 1) Vue du détecfeur de mouvement des mâchoires (A) ;

2) Mouton en cours de mesure avec l'appareil électronique : détecteur de mouvement (A), unité centrale de mesure (B). 

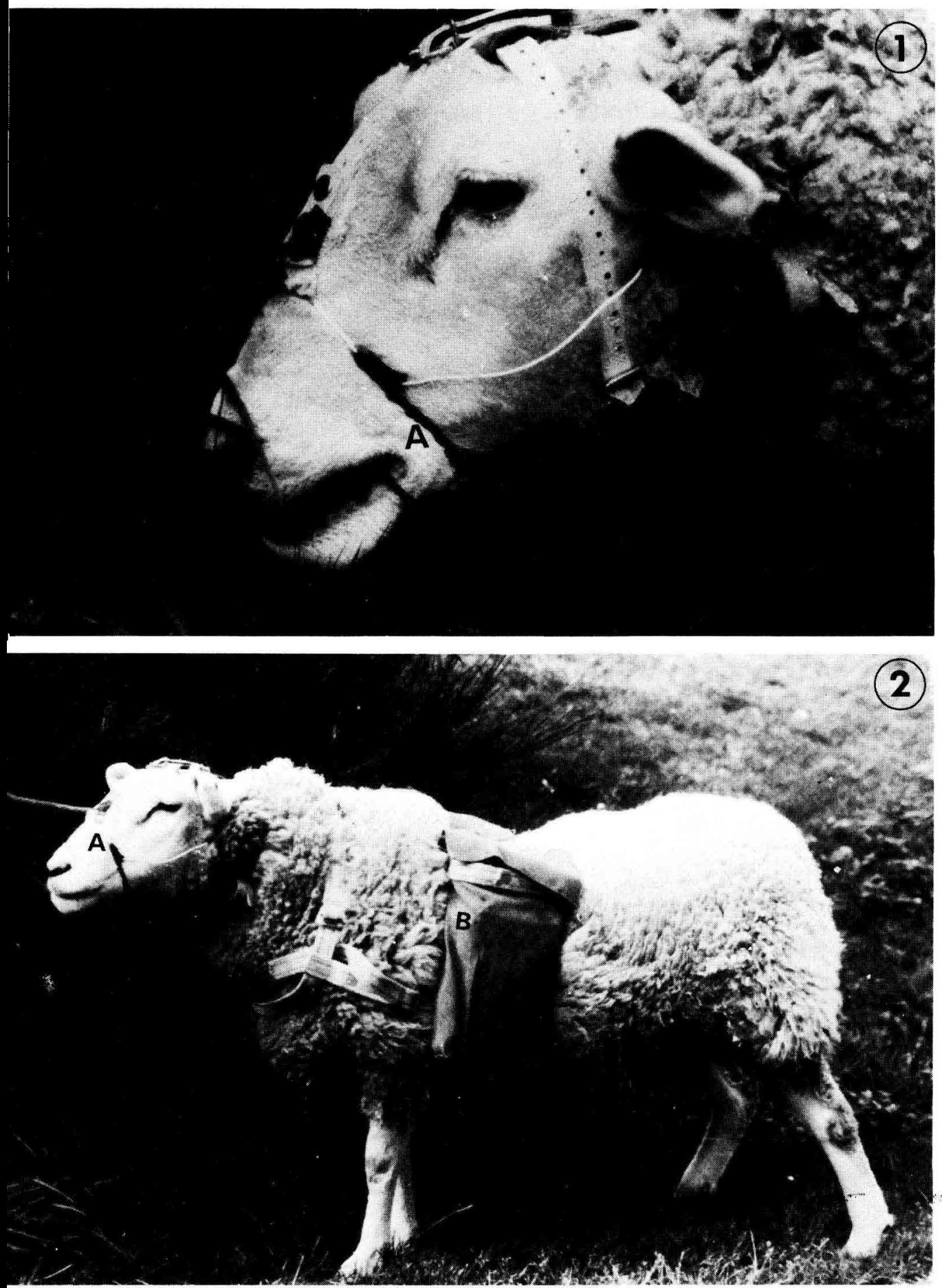
de suile. Les impulsions électriques identifiées par l'unité centrale sont alors dirigées vers des mémoires de stockage dont le contenu peut être connu à tout instant par affichage digital. Les données peuvent également être transcrites sur bande magnétique ou sur bande perforée lorsque les animaux sont maintenus en stabulation entravée.

L'énergie électrique nécessaire au fonctionnement de l'appareil est fournie par des piles ou des batteries. La puissance consommée, en dehors de l'utilisation des diodes électro-luminescentes, est de 360 milliwatts. Le montage électronique sur circuit imprimé est enfermé dans un boîtier en polychlorure de vinyle étanche et robuste. L'ensemble, dont les dimensions extérieures sont environ $20 \times 12 \times 5 \mathrm{~cm}$, pèse approximativement $500 \mathrm{~g}$. L'appareil est donc facilement porté par un mouton au pâturage à l'aide d'un bâł léger en toile de bâche maintenu en place par des harnais (fig. 2).

\section{Premiers résultats.}

Nous avons testé les performances de l'appareil en comparant les résultats obtenus par lecture directe de l'affichage digital avec ceux calculés par l'analyse d'un enregistrement graphique effectué simultanément pendant $24 \mathrm{~h}$, sur deux moutons, à l'aide d'un enregistreur multipiste.

Les résultats rapportés (tableau) montrent que l'écart maximal entre le nombre de coups de mâchoire mesuré el la valeur lue est de l'ordre de 1 p. 100 . Nous avons constaté que le nombre de coups de mâchoire affiché par l'appareil au cours des périodes de rumination esł généralement supérieur à celui réellement donné, alors que le nombre total de coups de mâchoire (rumination + ingestion) est identique pour les deux méthodes de mesure; le nombre de coups de mâchoire au cours de l'ingestion est donc compté par défaut. Ces différences s'expliquent car certaines phases d'ingestion peuvent être identifiées comme cycles de rumination si elles satisfont aux paramètres introduits dans l'unité centrale. En effet, l'observation d'enregistrements graphiques des mouvements de mâchoire permet de vérifier que les ruminants ingèrent parfois leurs aliments de manière discontinue. Leur comportement s'apparente ainsi à celui d'une rumination. Toutefois les erreurs restent limitées et sur une

TABLEAU

Comparaison des résultats obtenus respectivement par enregistrement graphique du comporfement alimentaire ef mérycique $\left({ }^{a}\right)$ ef par l'appareil électronique $\left({ }^{b}\right)$

\begin{tabular}{|c|c|c|}
\hline Animal & Mouton 1 & Mouton 2 \\
\hline Quantités de foin ingéré ( $\mathrm{g} M S / \mathrm{J}$ ) . . . & 1060 & 1180 \\
\hline $\begin{array}{l}\text { Nombre de coups de mâchoire (ingestion) } \begin{array}{l}\left({ }^{a}\right) \\
(b)\end{array} \\
\text { Erreur par défaut (p. 100).............. }\end{array}$ & $\begin{array}{l}8620 \\
8500 \\
1,39\end{array}$ & $\begin{array}{r}11550 \\
11460 \\
0,77\end{array}$ \\
\hline 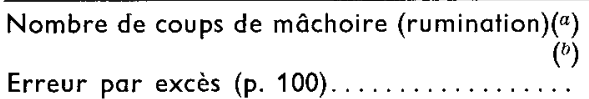 & $\begin{array}{l}11360 \\
11480 \\
\quad 1,05\end{array}$ & $\begin{array}{r}13480 \\
13570 \\
0,66\end{array}$ \\
\hline
\end{tabular}


période de mesure de $24 \mathrm{~h}$, nous avons constaté que les résultats donnés par l'appareil sont exacis à 1 p. 100 près. Ces premiers résultats devront être confirmés par une étude comparative effectuée sur un plus grand nombre d'animaux et avec une gamme de régimes plus étendue.

\section{Autres applications de l'appareil.}

L'unité centrale, qui possède déjà une horloge, peut être adaptée à la mesure de la durée respective des périodes d'ingestion, de rumination et de repos; il est également possible de déterminer l'heure du début et de la fin de chacune de ces périodes. On évite ainsi l'analyse manuelle des enregistrements graphiques des mouvements de mâchoire, qui est toujours fastidieuse, longue et donc source d'erreurs.

Le principe de détection proposé, basé sur l'exploitation d'un signal électrique et la mise en mémoire des informations permettent d'envisager l'exploitation à distance de ces dernières, à partir d'animaux au pâturage. La transmission des données peut se faire en un femps préalablement établi. Une forte puissance d'émission est ainsi disponible, ce qui permet d'accroître la distance entre émetteurs et récepteurs. II est aussi possible d'augmenter l'autonomie de l'appareillage ce qui limite au maximum les perturbations comportementales provoquées par la présence humaine.

Commission CNERNA Digestion-Absorption/Association des Physiologistes, Paris 5-6 octobre 1978.

\section{Références}

RUCKEBUSCH Y., 1963. Recherches sur lo régulation centrale du comportement alimentaire chez les ruminants. Thèse Doct. Sci. nat. Lyon, 213 pp.

RUCKEBUSCH Y., BUENO L., 1972. Analyse quantifative des relations activité alimentaire - état de vigilance chez les bovins en stabulation. Ann. Rech. vet., 3, 399-420.

RUCKEBUSCH Y., BUENO L., 1973. Un dispositif simple et aufonome d'enregistrement de l'activité alimentaire chez les bovins au pâturage. Ann. Rech. vét., 4, 627-636.

RUCKEBUSCH Y., BUENO L., LATOUR A., 1973. Recherches sur le comportement alimentaire des Ruminants. $X$ - Activité alimentaire des bovins au pâturage. Revue Méd. vét., 124, 685-691.

BECHET G., 1978. Enregistrement des activités alimentaires et méryciques des ovins au pâturage. Ann. Zootech., 27, 107-113. 